\title{
Contribution à l'étude des Zonitidae (Gastropoda) cavernicoles et endogés de Roumanie
}

\author{
par \\ Alexandrina NEGREA* \\ SUMMARY \\ Contributions to the study of the Zonitidae (Gastropoda) \\ cavernicolous and endogean from Rumania
}

This paper is a contribution to the study of the species of cavernicolous and endogean Zonitidae from Rumania. For each of the 24 species belonging to 6 genera we present the geographical distribution, their relation to the country's territory and data concerning the morphological characteristics, the genital apparatus included. The paper ends with a short faunistic, zoogeographical and ecological analysis.

Les Zonitidae sont des Gastéropodes à coquille aplatie, discoidale, luisante et transparente. Les espèces de Transylvanie ont constitué un objet d'étude dès 1863 (Bielz, Kimakowicz, etc), et en 1940 Boettger s'occupa aussi des espèces cavernicoles. Une première synthèse des connaissances sur la famille des Zonitidae de Roumanie apparait dans le volume Gastropoda Pulmonata (Grossu, 1955). Plus récemment, Negrea (1966) a publié une synthèse sur les Gastéropodes de nos grottes, les Zonitidae inclusivement. Une contribution à la connaissance des Zonitides roumaines des tribus Zonitini et Oxychilini est faite par Grossu et Riedel (1968) et spécialement sur le genre Oxychilus par Riedel (1969). Pinter (1972) fait une révision du genre Vitrea de Balkans, en se basant dans une certaine mesure aussi sur du matériel provenant de Roumanie.

D’après Riedel (1966), la famille des Zonitidae comprend 3 sous-familles, comme il suit:

Fam. Zonitidae Mörch, 1864

Sous-fam. Gastrodontinae Tryon, 1866

Sous-fam. Zonitinae Mörch, 1864 avec 3 tribus: Vitreini Thiele, 1931;Zonitini Mörch, 1864; Oxychilini Hesse in Geyer, 1927

Sous-fam. Daudebardiinae Kobelt, 1906

Les représentants de cette famille sont des éléments hygrophiles, vivant dans les forêts sous les feuilles mortes et dans le sol, sous les pierres, d'où ils peuvent pénétrer dans les grottes pendant les périodes sèches ou plus froides. Peu d'espèces sont troglophiles ou troglobies. Dans la zone directement éclairée et dans celle diffuse des grottes, elles rongent la microflore des parois humides.

\footnotetext{
* Institut de Spéologie “Emile Racovitza”, 9, rue Moxa, Bucarest 12, Roumanie.
} 
Dans ce travail nous nous occupons des représentants de la famille des Zonitidae, exception faite de ceux du genre Daudebardia plus rarement rencontrés dans les grottes. Dans la faune de notre pays on connait 6 genres.

\section{Sous-fam. Gastrodontinae \\ Gen. Zonitoides Lehmann, 1862}

Dans la zone paléarctique on connait 5 espèces de ce genre. En Europe et en Roumanie il est représenté par une seule espèce à répartition holarctique, Zonitoides nitidus.

La coquille à spire aplatie-conique, irrégulièrement striée, largement ombiliquée. Elle a 4-5 tours puissamment courbés.

\section{Zonitoides nitidus Müller 1774}

Espèce holarctique, troglophile. Elle vit dans les forêts, sur les bords des lacs et dans les prairies humides. En Roumanie elle est connue de quelques localités de Moldavie, Banat, Transylvanie et Dobrogea centrale.

Aux localités connues jusqu'à présent, nous ajoutons quelques grottes de Hunedoara: Pestera de sub Bulzul Hicionilor, Gaura lui Cocolbea et Gura Cetatii, et de Dobrogea: les forêts de Gura Dobrogei, Comana et Negru Voda.

Les exemplaires examinés par nous ont entre 4 et $6 \mathrm{~mm}$ de largeur. La coquille rouge-brun est formée de 4,5 - 4,7 tours; l'omibilic est large.

\section{Sous-fam. Zonitinae Trib. Vitreini Gen. Vitrea Fitzinger, 1833}

Vitrea fait partie des genres ouest-paléarctiques, son aire de distribution géographique étant comprise entre les limites suivantes: à l'Ouest, les iles situées à l'Est de l'Océan Atlantique et l'Islande; à l'Est, le Nord de l'Iran et le milieu de la partie européenne de l'U.R.S.S.; au Nord, la Scandinavie (jusqu'à $62^{\circ}-66^{\circ}$ ); au Sud, le Nord de l'Afrique, Israël, le Kurdistan irakien et Kopet-Dag (Riedel, 1966). En Roumanie il est représenté par 7 espèces et une problématique.

Les espèces de ce genre sont répandues surtout dans les régions karstiques, se trouvant sous les pierres, dans la mousse et dans les feuilles mortes (Pinter, 1972). Etant hygrophiles, nous les avons trouvées dans les forêts, dans les feuilles mortes et dans le sol sous ces feuilles, ainsi que sous les pierres d'où elle pénètrent souvent dans les grottes. Elles peuvent être rencontrées dans la zone éclairée directement ou dans celle diffuse des grottes, où elles rongent des biodermes d'Algues et de Bryophytes sur les parois humides.

La coquille est très petite, pouvant atteindre au maximum $6 \mathrm{~mm}$ de largeur. Elle a une forme discoïlale, le tour aplati et très mince. Colorée en nuances d'un blanc-jaunâtre, elle est souvent vitreuse, transparente. L'ouverture semi-lunaire est toujours verticale. 
Vitrea diaphana (Studer, 1820)

Espèce plutôt carpato-alpine, prédominante dans les Carpates, citée récemment par Pinter (1972) de Bulgarie et Yougoslavie. En Roumanie, cette espèce a été signalée de plusieurs localités, spécialement de monts Bihor, Fagaras, Piatra Craiului, Bucegi et le Nord des Carpates orientales.

Le matériel étudié par nous a été collecté dans les localité suivantes: Monts Apuseni: Circuitul Galbenei et Izvorul Vaii Stînei. Moldavie: Pîngarati, Rarau-la forêt Slatioara et Ilva Mare. Valachie: Sinaia (autour de la station zoologique), Poiana Cumpatu, Valea Alba et la vallée du Delghiu (Buzau).

Les exemplaires examinés ont une largeur de $4 \mathrm{~mm}$, une coquille avec 5,5 - 6 tours légèrement courbés; le dernier, vers l'ouverture est environ deux fois plus large par rapport, au pénultième.

\section{Vitrea transsylvanica (Clessin, 1877)}

Espèce prédominante dans les Carpates, signalée par Riedel (1966) et Pinter (1972) de Stara Planina (Bulgarie). Espèce probablement troglophile trouvée chez nous dans quelques grottes d'Olténie et de Transylvanie.

$V$. transsylvanica a été décrite par Clessin sur du matériel de Transylvanie. En Roumanie cette espèce est connue de beaucoup de localités des monts Apuseni, Carpates méridionales et orientales.

Nous l'avons identifiée dans le matériel collecté des grottes. Olténie: l'aven $\mathrm{n}^{\mathrm{O}} 2$ de Cazarmi, Pestera din Furca Vaii, Avenul de sub Plaiul Gorganului. Monts Persani: grotte $n^{\circ} 14$ des gorges du Virghis. Elle était présente aussi dans la litière de certaines forêts de Transylvanie (à l'entrée dans Pestera Gura Cetatii) et en Moldavie (la cabane Durau de Ceahlau).

Les exemplaires que nous avons examinés ont des dimensions un peu plus petites que celles indiquées par Pinter (1972), à savoir: 3,5-4 mm de largeur. La coquille est très finement et irrégulièrement striée, vitreuse et non ombiliquée. Les tours, au nombre de 4,5 - 5, sont beaucoup plus courbés, le dernier tour environ 3 fois plus large que le pénultième.

\section{Vitrea contracta (West., 1871)}

Espèce européenne, décrite de Suède. D'après Pinter (1972), elle est répandue partout en Europe, au Sud jusqu'au Nord de l'Afrique (Algérie, Libye) et en Crète, a l'Est jusqu'au Caucase et un Turquie. Son apparition dans les Açores et à Madère est problématique, selon l'auteur mentionne.'

Chez nous, cette espèce est citée de quelques localités de Transylvanie. Dans la collection de l'Institut de Spéleologie "E. Racovitza" nous avons examiné du matériel de la grotte $n^{0} 10$ de Valea Lupsei (Olténie) et Pestera Hotilor - Mangalia (Dobrogea), ainsi que dans l'endogé de certaines régions karstiques (litière de Valea Lupsei et de Bogata), comme dans la forêt Turuiac (Nord de la Dobrogea). Ces nouvelles localités complètent les données sur la répartition de l'espèce dans notre 
pays. On peut affirmer que son biotope préféré est constitué par les feuilles mortes humides des forêts. Pour les grottes elle est, probablement, trogloxène.

Espèce de petite taille, haute de $1,3 \mathrm{~mm}$ tout au plus et large de $2,5 \mathrm{~mm}$; la coquille incolore et transparente; 4 - 5 tours légèrement courbés, finement et irrégulièrement striés; le dernier tour 1,5 fois plus large que le pénultième; l'ombilic peu profond et relativement large; l'ouverture pointue, semi-lunaire.

Vitrea subcarinata (Clessin, 1877)

Espèce endémique dans les Carpates méridionales (oest de Transylvanie, Olténie, Banat). Pour le milieu cavernicole elle est citée de certaines grottes d'Olténie, Banat et Hunedoara (Negrea, 1960). Nous notons ici les localités certaines où nous avons du matériel pour cette espèce. Banat: Pestera Stîrnic. Hunedoara: Pestera Dutu et quelques stations endogées (la forêt entre la ligne de partage des eaux vers Fundatura Ponorului, l'insurgence Lunca Ponorului, des lithoclases sur la vallée Riù de Mori, lapidicole près de Pestera Cioclovina Uscata). Olténie: Pestera Muierilor, Pestera cu Gheața, Avenul de sub Plaiul Gorganului et la litière sur Valea Lupsei. Dans les endroits cités $V$. subcarinata préfère les lieux humides ayant beaucoup de matière organique (bois pourris). Il est possible que ce soit une espèce troglophile.

Les exemplaires examinés ont une taille jusqu'à 1,2 $\mathrm{mm}$ de hauteur et une largeur de 2,8 mm; la coquille luisante, à $5-6$ tours séparés par une suture profonde; l'ombilic mince et l'ouverture étroite, semi-lunaire.

\section{Vitrea crystallina Müller, 1774}

Espèce répandue dans presque toute l'Europe; dans la péninsule balkanique seulement en Yougoslavie et en Roumanie (Pinter, 1972). Elle vit dans les endroits humides, dans les feuilles mortes à humus, sous les pierres et les bois pourris. En Roumanie cette espèce est citée de plusieurs localités des Carpates orientales et méridionales, monts Apuseni et du plateau de Transylvanie; parmi celles-ci il y a aussi des grottes. Nous l'avons signalée dans un autre travail (Negrea, 1966) dans les grottes du bassin des vallées de Cerna et Berzeasca (Banat), les monts Persani et le sud de la Dobrogea, toujours en petit nombre, collectée sur l'argile et les feuilles mortes et la zone vestibulaire. Nous ajoutons deux stations endogées (la forêt Turuiac au nord de la Dobrogea et Baleia de Hunedoara).

Les exemplaires examinés ont une largeur de $2,1-4,0 \mathrm{~mm}$. La coquille finement et irrégulièrement striée, luisante, transparente; $4-5$ tours avec le dernier 1,5 fois plus large que le pénultième; l'ombilic mince.

Vitrea subrimata (Reinh., 1871)

Espèce alpino-méridionale (Zilch et Jaeckel, 1962). Pinter (1972) indique sa présence en Yougoslavie, Albanie, Bulgarie, Turquie, Chypre, Sicile, Malte, Sardaigne, Corse et Baléares. Elle est citée aussi par certains auteurs de Roumanie (Transylvanie). Negrea (1966) indique deux grottes du Banat (Pestera Zamonita et Pestera 
Gradinca). Nous ajoutons maintenant une autre grotte du Banat (Pestera Stîrnic) et deux grottes de Hunedoara (Pestera Gaura Oanei et Pestera de sub Vîrful Pîrjolii). Dans ces grottes elle vit dans des endroits à detritus végétal fin ou grossier. Elle est probablement trogloxène. Dans les stations endogées elle est apparue seulement dans Valea Draganului à Ciripa (monts Apuseni).

Assez semblable à Vitrea diaphana, Vitrea subrimata a une coquille à 4,5 - 5 tours modérément courbés et très peu relevés et l'ombilic assez mince. La coquille a une largeur de $2,5-3,5 \mathrm{~mm}$.

En dehors des espèces de Vitrea énumérées, retrouvées par nous dans les régions karstiques de Roumanie, Grossu (1955) et Pinter (1972) indiquent encore la présence de deux autres espèces de Vitrea. Il s'agit de Vitrea jetschini Kimak., collectée sur le mont Domogled (Baile Herculane) et de Vitrea jickelii Clessin, espèce probl: ematique, décrite de "Hunyad". Elles ne sont pas apparues dans le matériel de la zone respective examiné par nous.

\section{Gen. Troglovitrea Negrea et Riedel, 1968}

C'est un genre de la sous-famille Zonitinae, trib.Vitreini. Il a la coquille et la radula du type Vitrea et l'appareil génital du type Oxychilus. L'unique espèce connue de ce genre est T.argintarui.

\section{Troglovitrea argintarui Negrea et Riedel, 1968}

Espèce endémique pour les monts Mehedinti et les altitudes prémontagnardes qui, au Nord de son aire de respartition atteint la vallée supérieure de la Cerna, sans toutefois dépasser cette rivière vers l'Ouest. Elle se nourrit de plantes en décomposition et peur-être du guano des grottes. Espèce troglobie, qui possède des populations bien représentées dans plusieurs grottes: Pestera Closani, Pestera Vacilor din Steiul Orzestilor, l'aven au-dessus de Pestera Lazului, Pestera Cioaca cu Brebenei, les grottes $n^{\text {os }} 7$ et 14 de Valea Lupsei, les grottes $n^{\text {os }} 4,7,8$ de la vallée du Motru Sec et la grotte $n^{0} 21$ de la vallée de Cernisoara. Elle n'a pas été trouvée jusqu'à présent en dehors des grottes.

La coquille (fig.1, A) a les dimensions suivantes: la hauteur 2,1 mm, la largeur $4,4 \mathrm{~mm}$, le plus gros excemplaire atteint environ $5 \mathrm{~mm}$. Les tours au nombre de $41 / 2-42 / 3$, faiblement relevés, régulièrement ascendants, le dernier tour 2 fois plus large que la pénultième. L'ouverture légèrement oblique. La coquille de couleur blanchâtre ou jaune, lisse, non ombiliquée.

Le pénis gros, épais, plus long que le vagin considéré ensemble avec l'oviducte et se terminant par un fléau presque aussi gros que le pénis. L'épiphallus, épais et très court, s'ouvre dans le pénis latéralement. Le vagin assez long, à la base visiblement plus mince que le pénis. Le réceptacle séminal grand, ovale ou allongé en forme de demi-lune est fixé sur un court pédoncule (fig. 1, B). 

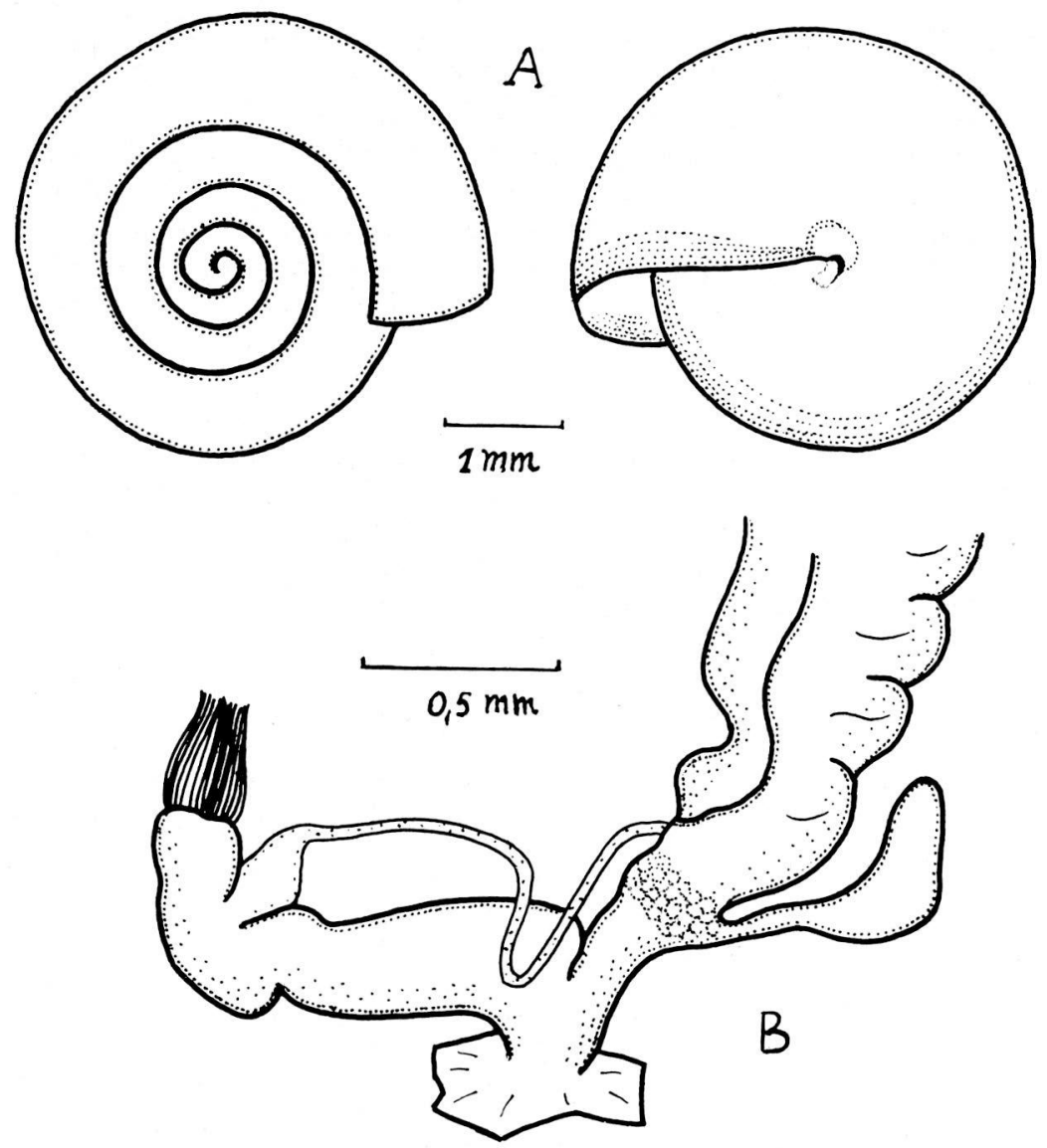

Fig. 1. Troglovitrea argintarui Negrea et Riedel, Pestera Closani. Coquille (A) et appareil génital (B) (orig.).

\section{Trib. Zonitini}

Gen. Aegopinella Lindholm, 1929

Ce genre, largement répandu en Europe, est représenté en Roumanie par 3 espèces. La coquille peut atteindre jusqu'à $16 \mathrm{~mm}$, a une spire très aplatie, irrégulièrement striée, avec une structure fine. Elle est formée de $4-4,5$ tours, le dernier vers l'ouverture de plus en plus dilaté et largement évasé. Toujours largement ombiliquée.

\section{Aegopinella minor (Stabile, 1864)}

Espèce sud-est européenne, fréquente en Bulgarie, Roumanie, Hongrie et Tchécoslo- 
vaquie; à l'Ouest elle s'étend jusqu'au Piémont, les Alpes et la Savoie (Forcart, 1959) et au Nord jusqu'à Bielowieza en Pologne et les environs de Novogroudok en Bielorussie (Riedel, 1957). Cette espèce provient probablement de Crimée et du Sud-Ouest du Caucase. Espèce probablement trogloxène. On la trouve dans des endroits chauds et relativement secs (forêts de chênes des plaines, régions calcaires, etc.). En Roumanie cette espèce est citée de quelques localités endogées des Carpartes orientales et méridionales et de la Dobrogea, ainsi que d'une grotte de Hunedoara (Cetatea Bolii).

Le matériel de A.minor de la collection de l'Institut de Spéologie provient autant de l'endogé que des grottes. Olténie: grotte $n^{0} 41$ de la vallée de la Cerna (Inelet); Pestera din Dealul Ruschiului; l'aven Gaura Mare; la litière des forêts de la vallée de la Lotrioara, la vallée du Lotru en aval de Voineasa; Crovul cu Gheața et Crovul de la Cazarmi. Banat: Pestera Gaura Pîrsului de la Capu Baciului; grotte no 2 de Cerveniaia et grotte $n^{0} 6$ de Cureacita; la litière des forêts de la vallée du Caras, les gorges de la Gîrlistea, la vallée de la Cerna et le défilé du Danube. Dobrogea: on l'a trouvée seulement dans les feuilles mortes des forêts de Caramat, Babadag, Murfatlar et Hagieni. Dans les monts Apuseni: une seule capture dans la forêt de hêtres de Valea Galbenei (Stîna de Vale).

Aegopinella minor est donc fréquente presque dans toute la Roumanie à l'exception des Carpates orientales où elle est assez rare. Dans cette région c'est l'espèce suivante qui est plus fréquente.

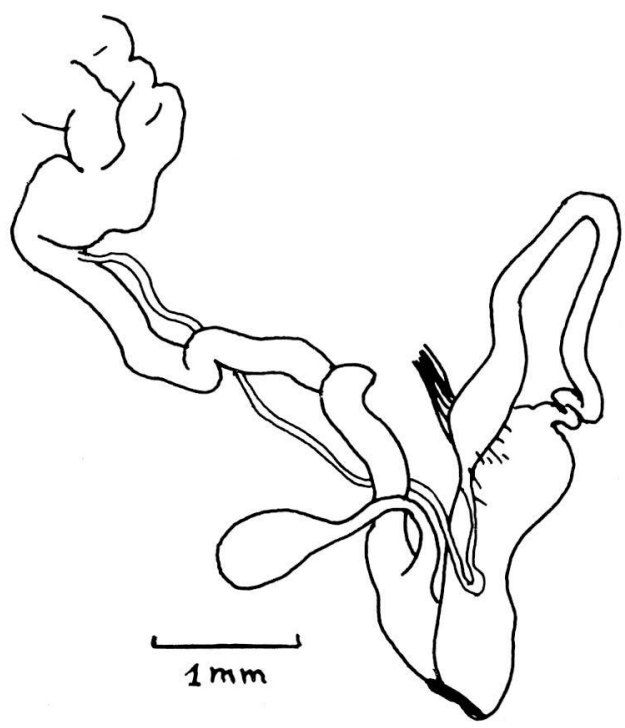

Fig. 2. Aegopinella minor (Stabile), Pestera Bolii. Appareil génital (d'après Grossu et Riedel, 1968). 
Les exemplaires que nous avons examinés ont jusq'à $7 \mathrm{~mm}$ de largeur, avec 4,5 tours, le dernier 1,8 fois plus large que le pénultième. La coquille est largement ombiliquée. Le pénis est épais et relativement court et l'épiphallus gros à la base. Le réceptacle séminal sphérique, à long pédoncule (fig. 2).

\section{Aegopinella epipedostoma (Fagot, 1879)}

Espèce connue du versant septentrional des Pyrénées jusque dans la région de Toulouse (France), des monts Taunus de l'Allemagne et des Sudètes tout au long des Carpates jusque dans la partie sud des Carpates orientales. Isolément elle est apparue dans les environs de Novogroudok (Biélorussie) (Riedel, 1957). Hudec (1964) différencie les populations carpatiques en une sous-espèce distincte, décrite de Tchécoslovaquie, Aegopinella epipedostoma iuncta (un paratype se trouve dans la collection du Musée d'Histoire Naturelle de Iassy).

En Roumanie elle est signalée par Husanu (1965) du Nord de la Moldavie (Putna - Radauti) comme nouvelle pour la faune du pays.

Grossu et Riedel (1968) donnent plusieurs localités des Carpates orientales où elle apparâit fréquemment et en grand nombre.

Nous la signalons dans la litière de certaines forêts de la vallée de la Prahova: Poiana Cumpatu (Sinaia) et Valea Alba (Busteni).

Les exemplaires de la vallée de la Prahova répondent exactement à la diagnose de l'espèce.

\section{Aegopinella pura (Alder, 1830)}

Elle peuple presque toute l'Europe. En Roumanie elle est fréquemment signalée des Carpates orientales (spécialement!), Carpates méridionales, monts Apuseni et le plateau de Transylvanie.

Nous ajoutons de nouvelles stations cavernicoles et endogées. Olténie: Closani, Valea Lupsei, le monastère Tismana, les gorges du Runcu. Banat: vallée de la Bîrzava, les gorges de la Gîrlistea; vallée de la Ponicova; vallée de la Cerna (Domogled). Hunedoara: Pestera Gaura Oanei, lithoclases près de Pestera Cioclovina Uscata. Mcnts Persani: les gorges du Vîrghis. Monts Apuseni: forêt de Valea Stînei. Valachie: forêt de la vallée du Buzau, étant la première citée pour cette province. Espèce trogloxène.

La coquille a une largeur allant jusqu'à 3,5 mm et 3,5 tours, le dernier étant plus aplati et 1,5 fois plus large que le pénultième. L'ombilic est large.

Gen. Nesovitrea Cooke, 1921

Nesovitrea possède 2 sous-genres: Nesovitrea s.str., répandu dans les iles Hawaii, et Perpolita Baker, répandu dans les régions holarctiques et néotropicales. En Roumenie on connait 2 espèces: $N$.hammonis et $N$.petronella, la dernière citée récemment par Grossu et Riedel (1968).

La coquille est petite, entre 3 et $8 \mathrm{~mm}$ (chez Perpolita seulement jusqu'à 5,50 $\mathrm{mm})$; elle a $3,5-4,4$ tours. 


\section{Nesovitrea (Perpolita) hammonis (Ström, 1765)}

C'est une des plus répandues espèces paléarctiques, d'Islande et des Açores jusqu'au Kamtchatka, les Kouriles et la Corée (Grossu et Riedel 1968). En Europe elle est plus fréquente dans la partie centrale et méridionale, rare dans le Midi et absente dans les pays méditerranéens. Boettger (1940) la signale sous le nom de Retinella (R.) hammonis de Valea Huzi et Valea Stearpa près d'Albac (monts Apuseni). Grossu et Riedel (1968) mentionnent les localités: Tarcau (Neamt), Rasinari (Sibiu), le refuge Postavarul et Sinaia.

Nous la signalons en plus de Pî̀iul Vadului (Cluj). Par conséquent, chez nous elle vit dans la zone montagneuse et sous-carpatique, étant très rare dans le reste du pays.

Les exemplaires examinés ont 3,8 mm de largeur, avec 3,5 tours, le dernier 2 fois plus large que le pénultième.

\section{Nesovitrea (Perpolita) petronella (L.Pfeiffer, 1853)}

Espèce boréo-montagnarde rare dans les Carpates. Grossu et Riedel (1968) la mentionnent comme une nouvelle espèce pour la Roumanie, sur la base des exemplaires collectés non loin de Gheorghieni, dans les monts Haghimasu Mare, et de l'exemplaire collecté par W. Polinski sur les Jepii Mari (monts Bucegi). Dans le matériel des zones karstiques examiné par nous, nous ne l'avons pas trouvée.

\section{Trib. Oxychilini \\ Gen Oxychilius Fitzinger, 1833}

Ce genre a une répartition ouest-paléarctique, étant représenté en Roumanie par 9 espèces (Grossu et Riedel, 1968) parmi lesquelles 4 seulement ont été rencontrées par nous dans les grottes et dans l'endogé.

La coquille a des dimensions moyenne ( $8-21 \mathrm{~mm}$ de largeur), les tours irrégulièrement striés ou lisses, luisants. Elle a $5-6,75$ tours (rarement seulement 4,5). Le dernier tour vers l'ouverture non évasé, mais souvent entièrement élargi. L'ombilic, toujours présent, est variable, mais jamais perspectif.

\section{Oxychilus (Longiphallus) deilus malinowskii (L. Pfeiffer, 1865)}

Sous-espèce endémique au Nord de la Dobrogea. Grossu et Riedel (1968) indiquent les localités suivantes: Jijila, Luncavita, Niculitel et Cocosu. Bien qu'on eût collecté du matériel dans ces localités, elle manquait ce qui fait qu'on peut penser qu'elle est, probablement, assez rare.

La coquille, grande, atteint jusqu'à $21 \mathrm{~mm}$ de largeur et $16-19 \mathrm{~mm}$ de hauteur. Le dernier tour est 2 fois plus large que le pénultième. Le pénis cylindrique, au muscle rétracteur mince et long. L'épiphallus, court et mince, s'ouvre à $1 / 3$ du bout du pénis. Le réceptacle séminal allongé et étroit (fig.3). 


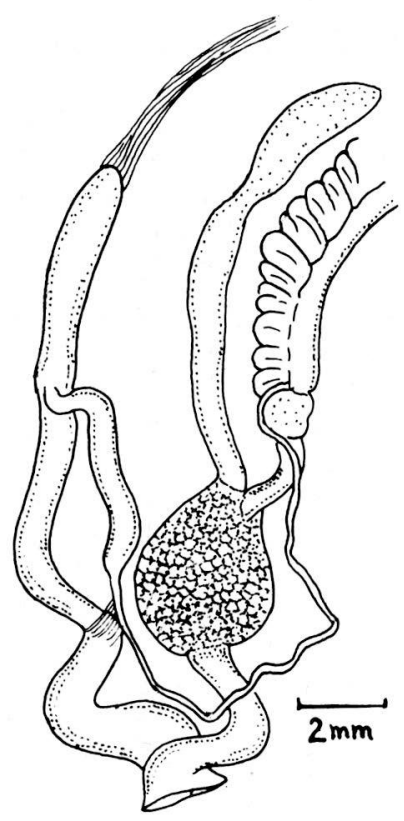

Fig. 3. Oxychilus (L.) deilus malinowskii (L.P.), nord de la Dobrogea. Appareil génital et coquille (d'après Grossu et Riedel, 1958).
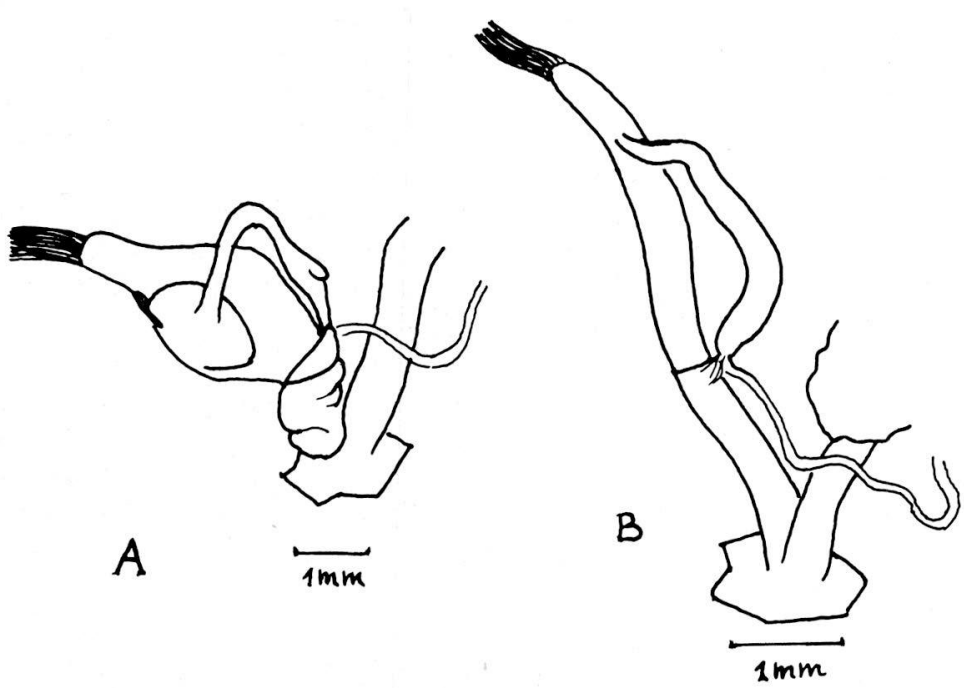

Fig. 4. Oxychilus (C) orientalis (Clessin), Codlea, appareil génital ơ (A) et Oxychilus (O.) cellarius (Müller), Westfalen-Lengerich, appareil génital ơ (B) (d'après Grossu et Riedel, 1968). 


\section{Oxychilus (Cellariopsis) orientalis (Clessin, 1887)}

Espèce carpatique décrite des Tatras et répandue dans les Carpates septentrionales et orientales. En Roumanie elle est connue dans les Carpates orientales et leurs Sous-Carpates, des monts Rodnei et Suceava jusqu'aux monts Bucegi et Piatra Craiului. Grossu et Riedel (1968) ont montré que les autres localités de Transylvanie et des Carpates méridionales, à l'Ouest de l'Olt, sont incertaines.

Nous la signalons dans Pestera de la Sugau (Carpates orientales), d'où nous possédons un exemplaire juvénile de $3 \mathrm{~mm}$ de largeur (leg.M.Dumitresco).

La coquille est très petite, pouvant atteindre $9-11 \mathrm{~mm}$ de largeur. La spire aplatie, très mince, de couleur blanche. Vu que d'après sa coquille cette espèce pourrait être confondue avec Oxychilus cellarius (Müller), il est très important d'examiner son appareil génital, particulièrement le pénis et l'épiphallus. Chez O.orientalis le pénis est gros et enflé et l'épiphallus muni d'un diverticule au bout, tandis que chez $O$. cellarius le pénis est cylindrique et mince et l'épiphallus simple (fig. 4.)

Oxychilus (Oxychilus) cellarius (Müller, 1774)

Zilch et Jaeckel (1962) la présentent comme un espèce européenne sous-atlantique, et Grossu et Riedel (1968) la citent du centre et de l'Ouest de l'Europe. Les derniers auteurs montrent que O.cellarius a été citée de beaucoup de localités de Roumanie (Carpates orientales, hauts plateaux de Transylvanie et plaine Roumanie), mais que ces localités sont incertaines. Probablement, les exemplaires dont il est question appartiennent à d'autres espèces avec lesquelles elle peut être confondue d'après sa coquille et on propose que O.cellarius soit éliminée pour le moment de la liste de la faune roumaine. Pourtant on ne peut exclure la possibilité d'apparition de cette espèce en Roumanie comme élément synanthrope, apporté par transport de l'Ouest de l'Europe. En ce qui nous concerne, nous ne l'avons jamais trouvée dans les zones karstiques.

\section{Oxychilus (Oxychilus) draparnaudi (Beck, 1837)}

Espèce ouest- et sud-européenne. Pour la Roumanie elle est donnée comme certaine par Grossu et Riedel (1968) seulement pour les jardins de Sibiu et de Cluj. Comme il s'agit de "biotopes synanthropes", les auteurs supposent qu'elle provient de transports des pays voisins. Nous ne l'avons pas rencontrée dans le matériel étudié.

La coquille est plus grande que chez O.cellarius et chez O.orientalis; la spire est aplatie, irrégulièrement striée, et semble plutôt lisse. Elle a 5,75 tours, le dernier plus dilaté à la périphérie. L'ombilic en quelque sorte large. Anatomiquement elle diffère de $O$. cellarius par un pénis et un épiphallus plus longs (fig. 5).

Oxychilus (Oxychilus) hydatinus (Rossmaessler, 1838)

Espèce circumméditerranéenne, dont l'aire de dispersion s'étend d'Espagne jusqu'à 


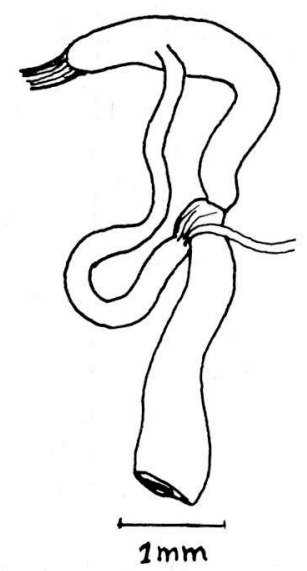

Fig. 5. Oxychilus (O.) draparnaudi (Beck), Sibiu. Appareil génital (d'après Grossu et Riedel, 1968).

l'Ouest de l'Asie Mineure. Fréquente dans les pays balkaniques du Midi, plus rare dans ceux du Nord, elle a été cependant identifiée en Croatie, Istrie et le Nord-Est de la Bulgarie (Riedel, 1962). En 1927 Samsonowicz a trouvé une coquille typique dans les calcaires tertiaires de la vallée Kara-Su, à l'ouest de Constanta (Grossu et Riedel, 1968). On suppose que sa rareté est due au fait qu'elle vit profondément enfouie dans le sol. Nous ne l'avons pas trouvée dans le matériel collecté en Dobrogea.

\section{Oxychilus (Schistophallus) oscari (Kimakowicz, 1883)}

Kimakowicz $(1833,1890)$ décrit cette espèce de Bogata et de quelques autres localités des monts Persani, en décrivant aussi une "var.tumida" de Codlea (Transylvanie). Elle a été signalée aussi au SE de la Bulgarie (Riedel, 1961). En 1967 Riedel retrouve O.oscari dans "terra typica", c'est-à-dire à Bogata et Codlea (Grossu et Riedel, 1968). Les exemplaires vivants trouvés dans les fentes des roches peuvent être confondus avec O.glaber, toutefois ils en diffèrent par les lignes évidentes en spirale, sur la coquille. Une révision de la description de cette espèce est faite par Riedel (1972). Nous n'avons trouvé dans aucune localité des exemplaires de $O$. oscari.

\section{Oxychilus (Morlina) glaber (Rossmaessler, 1835)}

Espèce troglophile, répandue en Europe centrale et méridionale. La Roumanie se trouve dans l'aire de dispersion de la sous-espèce $O$. glaber striarius (West.) (Riedel, 1969). Elle peuple tout le pays, se trouvant autant dans la montagne que sur les collines, le plus fréquemment dans les grottes d'Olténie et du Banat. Il est intéres- 
sant de montrer que $O$. glaber striarus a été décrite sur un matériel de Sibiu comme Zonites (Hyalina) glaber var. striaria West. 1881.

De toutes les provinces de Roumanie, c'est en Olténie que O.glaber est le mieux représentée. Nous pourrions citer des dizaines de grottes des monts Vilcan et la région Runcu - Tismana, les monts et le plateau Mehedinti et le bassin supérieur de la Cerna.

En Banat nous l'avons trouvée dans un assez grand nombre de grottes dans: le bassin inférieur de la Cerna, la zone calcaire des monts Locva (très fréquente), les gorges de la Nera, le bassin des vallées Minis et Lapusnic, les gorges du Caras et les monts Doman. De Transylvanie nous avons des exemplaires de Pestera de sub Bulzul Hicionilor (bassin de Jiu de 1'ouest, Hunedoara) et de Pestera de la Sugau (monts Gurghiu - Harghita) et de quelques grottes des monts Persani et Apuseni. Dans les grottes de la Dobrogea elle est apparue plus rarement: Pestera Gura Dobrogei, l'aven de Cheia et Pestera de la Limanu. Dans la collection nous disposons encore de matériel de O.glaber collecté dans la litière de nombreuses forêts des zones karstiques mentionnées, aussi bien que des zones non karstiques de Transylvanie, Banat, Olténie, Dobrogea et Moldavie.

Selon Vandel (1964), les espèces de Oxychilus sont polyphages, se nourrissant de détritus végétal et animal. Gueorguiev et Beron (1962) considèrent O.glaber comme un élément guanophile et adéphage, consommant les larves de certains Diptères guanophiles. Motas, Decou et Burghele (1967) montrent que dans les grottes d'Olténie elles se nourrissent d'exuvies de Meta menardi. D'après nos observations (Negrea, Negrea 1972) il résulte que O. glaber est une espèce polyphage qui vit dans les grottes du Banat, simultanément ou à différentes dates, sur des substratums différents. Ainsi, d'un total de 31 grottes, nous l'avons trouvée dans 11 grottes seulement sur le plancher recouvert d'argile et de détritus végétal, sur des bois pourris ou sur le sable; dans 9 grottes seulement sur les parois; dans 7 grottes sur les parois et sur le plancher sans guano; dans 3 grottes sur les parois et le guano; dans une seule grotte on les a trouvées sur les parois et le plancher, sur le guano inclusive-
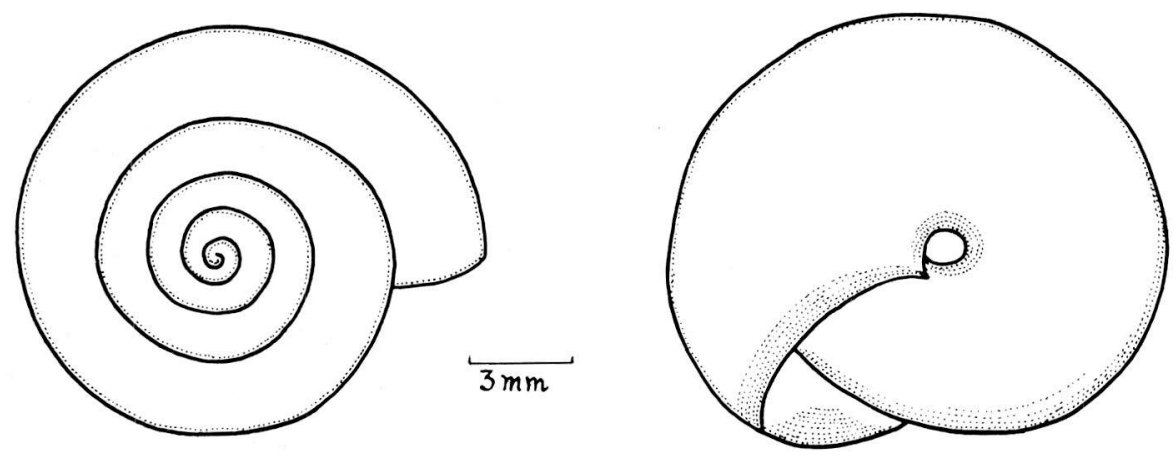

Fig. 6. Oxychilus (M.) glaber (Rossm.), Pestera Iasînoca. Coquille (orig.) 
ment. Nous pouvons affirmer ne pas avoir constaté une relation trophique évidente entre $O$. glaber et le guano. Dans ce sens nous remarquons qu'elle manque dans les grandes grottes à guano abondant (Pestera Dubova et Pestera Liliecilor), étant toujours présente dans les grottes plus petites, sans guano, et dans leurs environs.

Toujours sur la base de nos observations faites dans les grottes du Banat, il est intéressant de montrer que les individus peuplent la zone de l'entrée de la grotte jusqu'à $150 \mathrm{~m}$ de profondeur, préférant les galeris à lumière diffuse et le commencement de celles non éclairées. Les endroits des grottes où les individus se maintiennent toute l'année, formant parfois des agglomérations de dizaines d'exemplaires, manquent de courants d'air, l'humidité étant dans la majorité des cas proche de $100 \%$ et la température variant entre 11 et $16^{\circ} \mathrm{C}$.

La coquille (fig. 6), ayant une largeur de $10-14 \mathrm{~mm}$, est très luisante et faiblement striée. Elle présente $5-5,25$ tours, le dernier un peu plus large que le pénultième. L'ombilic est petit. Au point de vue de l'anatomie, $O$. glaber diffère des autres espèces du genre par la constitution interne du pénis. L'appareil génital (fig.7) est caractérisé par un pénis allongé, plus enflé dans la zone de l'ouverture. L'épiphallus, lui aussi est assez allongé. Le réceptacle séminal est bien délimité par le pédoncule long, de forme ellipsoidale.

\section{Oxychilus (Riedelius) depressus (Sterki, 1880)}

Espèce carpato-alpine à répartition méridionale jusque dans les pays du Nord de la péninsule balkanique. En Roumanie est répandue dans la partie montagneuse du pays, les populations étant très peu abondantes: plusieurs localités endogées des Carpates orientales et méridionales, en commençant par les monts Rodna jusqu'aux monts Cerna et certaines grottes du Transylvanie, Olténie et Banat; espèce troglophile.

Le matériel cavernicole dont nous disposons provient de plusieurs grottes réparties ainsi: Olténie: Pestera Polovragi, Pestera de la Ponoare, les grottes $n^{\text {os }} 6,8,11$, 12, 16 de Valea Lupsei, Pestera Vacilor de Closani, la grotte ${ }^{\circ} 5$ de Closani, Pestera Lazului, Pestera din Crovul cu Feriga de Marcoane, la grotte $n^{0} 1$ de Valea Bulbei, Pestera de La Pietre, Pestera Hotilor - Nadanova, Pestera Sfoadei, Pestera Topolnita (secteur Sohodol), grottes $n^{o s} 1,3,8,15,17,21$ de la vallée de la Cernisoara, grotte $\mathrm{n}^{0} 41$ de la vallée de la Cerna (Inelet). Banat: Pestera Comarnic, Pestera Socolovat, Pestera $n^{\circ} 2$ de sub Crno Pole, Pestera de sub Padina Popii, Pestera din Cleantul Zbegului, Pestera Gura Ponicovei. Hunedoara: Pestera Gura Cetatii. Monts Padurea Craiului (Apuseni): Pestera Vacii, Pestera Preguz, Pestera de la Izvorul Toplitei, Pestera de la Galasesti. Monts Trascau (Apuseni): Pestera din Valea Ghibartului. Monts Bucegi: Pestera Mica din Cheile Tatarului.

La coquille (fig.8) a une épaisseur de $8-10 \mathrm{~mm}$, le tour est très peu relevé, légèrement strié et luisant. Elle présente $4,5-5$ tours, le dernier 1,5 fois plus large que le pénultième. L'ombilic est très mince. Par comparaison avec 0 . glaber, la coquille est plus petite et plus aplatie.

Le pénis est gonflé, bien des fois presque sphérique, le muscle long et habituellement mince. L'épiphallus court et épais s'ouvre dans le pénis presque latéralement. 


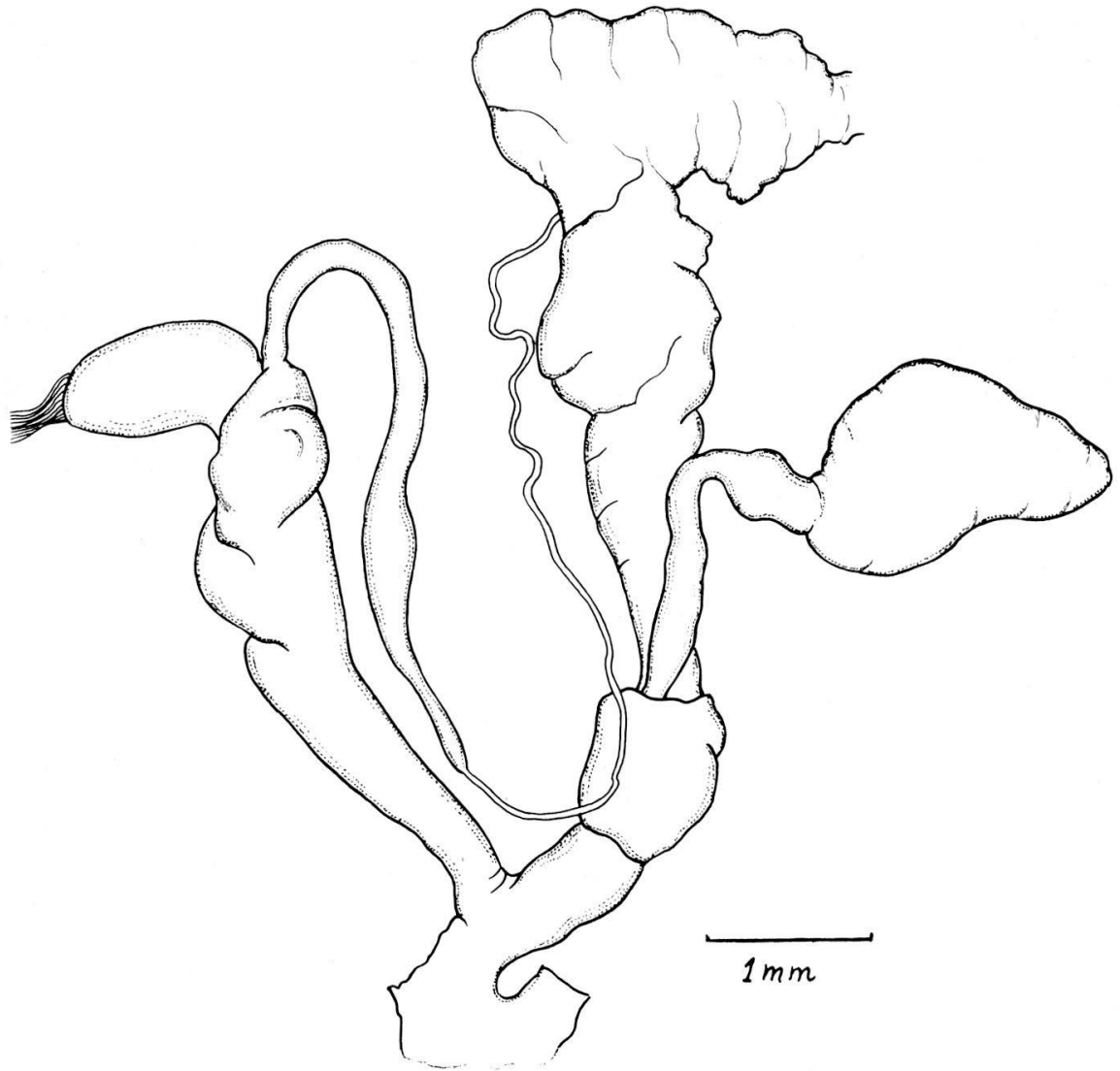

Fig. 7. Oxychilus (M.) glaber (Rossm.), Pestera Vraska. Appareil génital (orig.)
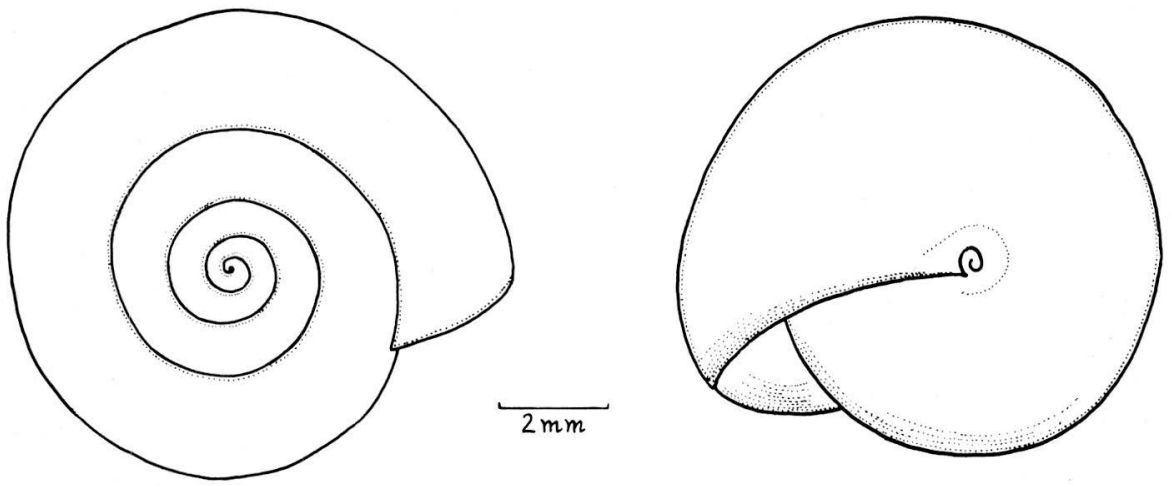

Fig. 8. Oxychilus (R.) depressus (Sterki), Pestera Ponoare. Coquille (orig.) 
Le vagin et l'oviducte ont à peu près la même grosseur. Le réceptacle séminal est bien délimité, de forme sphérique ou ovale. Ce qui est caractéristique c'est que les oviductes contournent le vagin (fig.9).

\section{Oxychilus (Riedelius) inopinatus (Ulicny, 1887)}

Espèce balkano-sous-carpatique (? ); elle s'étend à l'Ouest jusque près des monts Hert de Tchécoslovaquie, au Nord jusque près de Wloclawek en Pologne (Grossu et Riedel, 1968). Elle vit dans des endroits secs, de préférence dans des régions calcaires, bien enfoncée dans le sol.

En Roumanie elle a été signalée jusqu’à présent de quelques localités de Transylvanie et d'une station d'Olténie (Grossu et Riedel, 1968).

Dans le matériel que nous avons étudié elle n'a pas été identifiée.

\section{Oxychilus (Riedelius) montivagus (Kimakowicz, 1890)}

Espèce sous-carpatique endémique, répandue dans les Carpates entre le défilé de l'Olt et le Danube (aus Sud) et les monts Apuseni (au Nord).

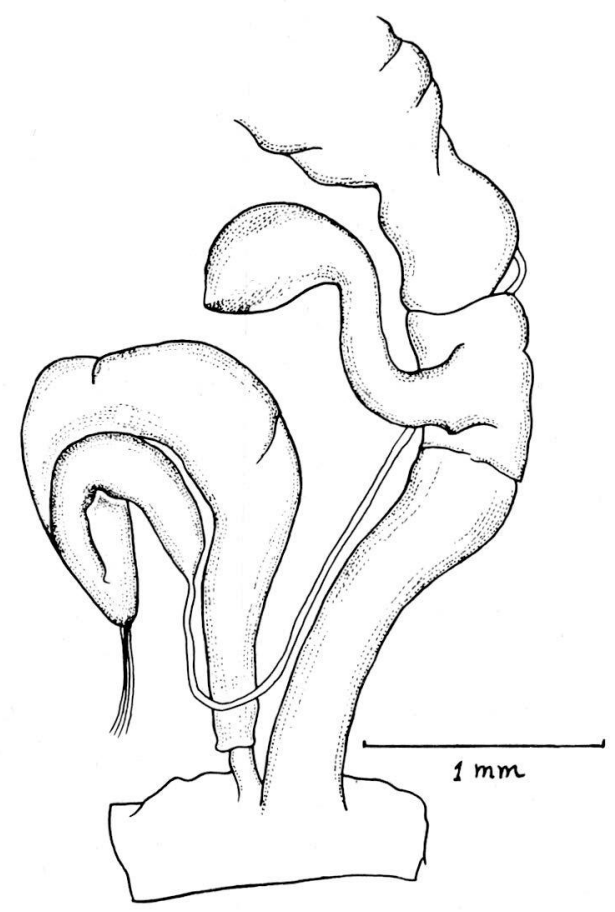

Fig. 9. Oxychilus (R.) depressus (Sterki), grotte $\mathrm{n}^{\mathrm{O}} 8$ de Valea Lupsei. Appareil génital (orig.) 

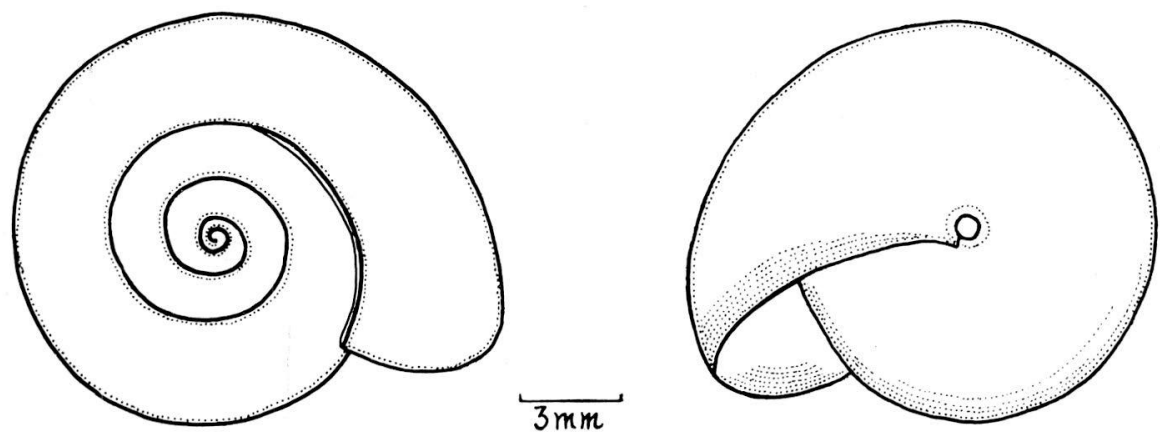

Fig. 10. Oxychilus montivagus (Kim.), Pestera Gaura Haiduceasca. Coquille. (orig.)

Nous la signalons de quelques grottes du Banat: Pestera cu Oase de Stîrnic, Pestera din Drumul Prolazului, Pestera $n^{0} 2$ de sub Crno Pole et Pestera Gaura Haiduceasca (fréquente) et de Padurea Craiului (Apuseni): Pestera de la Calatea et dans la litière sur Valea Draganului. Espèce troglophile.

La coquille (fig.10) a 10 - $14 \mathrm{~mm}$. Elle est finement striée et luisante, formée de $4,5-5$ tours, le dernier étant 2,5 fois plus large que le pénultième.

Le pénis est épais et puissant. L'épiphallus s'ouvre latéralement, près de l'apex du pénis et du fléau de celui-ci. Il est mince, plus court que le pénis. Le canal déférent long. Le réceptacle séminal est étroit et très allongé, pointu au bout et le pédoncule plus court (fig.11).

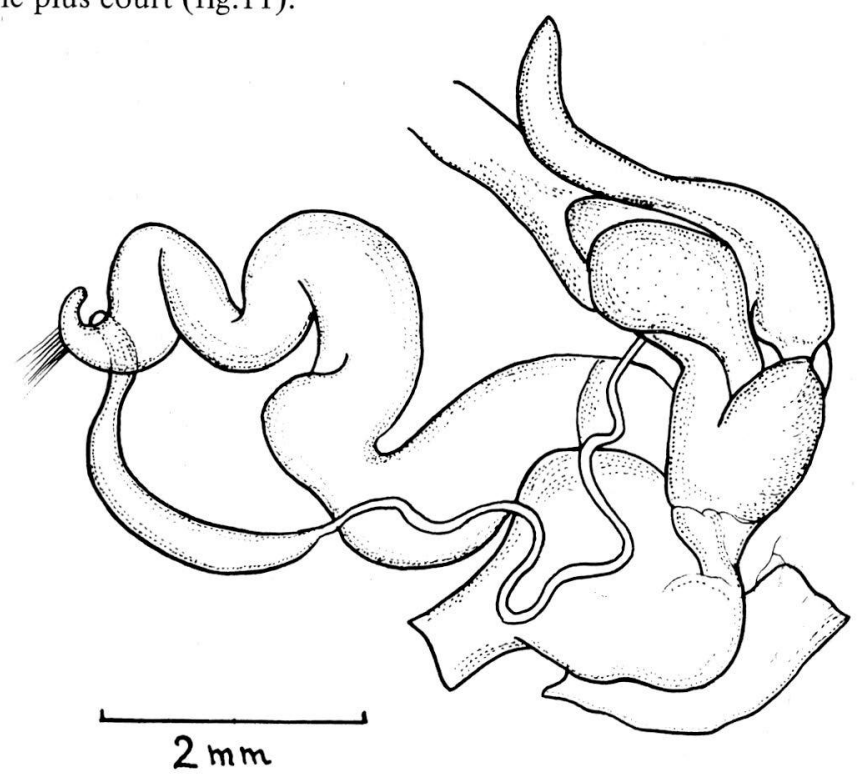

Fig. 11. Oxychilus montivagus (Kim.) Pestera Gaura Haiduceasca. Appareil génital (orig.) 
En nous référant au 3 espèces d' Oxychilus les mieux représentées dans la faune de notre pays $(O$. glaber, $O$. depressus et $O$. montivagus), nous constatons qu'habituellement leurs populations ne cohabitent pas dans les grottes d'Olténie et du Banat. Pourtant, nous avons remarqué aussi des cas de cohabitation. Ainsi, $O$. glaber et $O$. depressus vivent ensemble dans les grottes suivantes d'Olténie: les grottes $n^{\text {os }} 11$, 12, 16 de Valea Lupsei et Pestera din Crovul cu Feriga de Marcoane. En ce qui concerne $O$. glaber et $O$. montivagus, nous les avons trouvées ensemble seulement dans Pestera Gaura Haiduceasca du Banat.

\section{CONCLUSIONS}

En 1968 Grossu et Riedel on fait une révision systématique des espèces de Zonitidae de Roumanie, fondée sur l'étude de l'appareil génital. C'est ce qui nous a déterminé de procéder à une révision de tout le matériel de Zonitidae de la collection de l'Institut de Spéologie ,Emile Racovitza” y compris celui collecté par nous ces dernières années*. Les résultats obtenus, présentés dans ce travail, démontrent que dans les grottes et l'endogé de notre pays vivent 24 espèces, appartenant à 6 genres.

Par rapport à nos travaux antérieurs (Negrea, 1963, 1965, 1966), la liste des espèces de Zonitidae s'est enrichie de 11 espèces, à savoir: une espèce troglobie (Troglovitrea argintarui); 3 espèces des grottes et de l'endogé des zones karstiques (Vitrea contracta, Aegopinella minor et Oxychilus orientalis); 3 espèces de l'endogé karstique (Aegopinella epipedostoma, Oxychilus inopinatus et O. hydatinus); 3 espèces des zones non karstiques (Nesovitrea hammonis, $N$. petronella et Oxychilus deilus malinowskii); enfin, une espèce des biotopes synanthropes, Oxychilus draparnaudi. Pour les espèces déjà mentionnées dans les travaux cités, l'aire de dispersion geographique est complétée par l'indication de nouvelles stations cavernicoles ou endogées de zones déjà connues ou même de nouvelles régions.

La faune de Zonitidae de Roumenie est remarquable par les espèces endémiques qu'elle contient. Les unes occupent une aire restreinte à certaines zones des Carpates (Troglovitrea argintarui, Oxychilus montivagus, O. oscari et Vitrea subcarinata), d'autres au Nord de la Dobrogea (Oxychilus deilus malinowskii). Parmi les espèces carpatiques nous mentionnons Vitrea transsylvanica et Oxychilus orientalis. Les espèces $O$. depressus et Vitrea diaphana sont carpato-alpines et $O$. inopinatus, probablement balkano-sous-carpatique. Plusieurs espéces ont une répartition limitée à certaines parites de l'Europe: Nesovitrea petronella, Vitrea subrimata, Aegopinella minor, Oxychilus glaber, O. draparnaudi et Aegopinella epipedostoma. Trois es-

\footnotetext{
* Nous remercions, par cette voie aussi Monsieur le Docteur Adolf Riedel (Varsovie) pour son amabilité à vérifier la majorité de nos déterminations de Zonitidae de la collection, aussi que pour ses suggestions d'améliorer le tex te de ce travail. Nous remercions par cette voie, aussi les chercheurs de l'Institut de Spéologie "Emile Racovitza" pour le matériel collecté et mis à notre disposition.
} 
pèces ont une répartition vaste européenne (Vitrea contracta, V. crystallina et Aegopinella pura), une espèce est circumméditeranéene (Oxychilus hydatinus), une autre paléarctique (Nesovitrea hammonis) et une holarctique (Zonitoides nitidus).

$\mathrm{Au}$ point de vue écologique, la plus intéressante, sans doute, est l'espèce endémique troglobie Troglovitrea argintarui, limitée strictement aux grottes du Nord-Ouest d'Olténie. En dehors de celle-ci dans les grottes de Roumanie vivent encore quatre espèces troglophiles (Oxychilus glaber, O. depressus, O. montivagus et Zonitoides nitidus) et trois apparemment troglophiles (Vitrea subcarinata, V. transsylvanica et Acgopinella minor). Il existe encore 6 espèces trogloxènes, à savoir: Vitrea diapha$n a, V$. contracta, V. crystallina, V. subrimata, Aegopinella pura et Oxychilus orientalis.

Tableau 1. Fréquence des espèces de Zonitidae des grottes de la Roumanie pour les 5 provinces biospéologiques.

\begin{tabular}{|c|c|c|c|c|c|c|c|c|c|c|}
\hline Provinces biospéologiques & \multicolumn{2}{|c|}{ I } & \multicolumn{2}{|c|}{ II } & \multicolumn{2}{|c|}{ III } & \multicolumn{2}{|c|}{ IV } & \multicolumn{2}{|c|}{$\mathrm{V}$} \\
\hline $\mathrm{N}^{\mathrm{O}}$ total des grottes à Zonitidae & \multicolumn{2}{|c|}{3} & \multicolumn{2}{|c|}{81} & \multicolumn{2}{|c|}{32} & \multicolumn{2}{|c|}{16} & \multicolumn{2}{|c|}{4} \\
\hline $\mathrm{N}^{\mathrm{O}}$ des grottes à Zonitidae, \% & $n^{\circ}$ & $\%$ & $\mathrm{n}^{\mathrm{o}}$ & $\%$ & $n^{o}$ & $\%$ & $n^{o}$ & $\%$ & $n^{o}$ & $\%$ \\
\hline 1. Zonitoides nitidus & - & - & 3 & 3,7 & - & - & - & - & - & - \\
\hline 2. Aegopinella minor & - & - & 4 & 4,9 & 3 & 9,3 & - & - & - & - \\
\hline 3. Aegopinella pura & - & - & 1 & 1,2 & - & - & - & - & -- & - \\
\hline 4. Oxychilus orientalis & 1 & 33 & - & - & - & - & - & - & - & - \\
\hline 5. Oxychilus glaber & 1 & 33 & 48 & 59,2 & 22 & 68,7 & - & - & 3 & 75 \\
\hline 6. Oxychilus depressus & - & - & 16 & 19,7 & 6 & 18,7 & 9 & 56,2 & - & - \\
\hline 7. Oxychilus montivagus & - & - & - & - & 4 & 12,5 & 1 & 6,2 & - & - \\
\hline 8. Vitrea diaphana & - & - & - & - & - & - & 1 & 6,2 & - & - \\
\hline 9. Vitrea transsylvanica & 1 & 33 & 3 & 3,7 & - & - & 2 & 12,5 & - & - \\
\hline 10. Vitrea contracta & - & - & 1 & 1,2 & - & - & - & - & 1 & 25 \\
\hline 11. Vitrea subcarinata & - & - & 5 & 6,1 & 1 & 3,1 & - & - & - & - \\
\hline 12. Vitrea crystallina & 1 & 33 & 1 & 1,2 & - & - & 2 & 12,5 & - & - \\
\hline 13. Vitrea subrimata & - & - & 2 & 2,4 & 3 & 9,3 & - & - & - & - \\
\hline 14. Troglovitrea argintarui & - & - & 10 & 12,3 & - & - & - & - & - & - \\
\hline
\end{tabular}


Tableau 2. Diagramme de la cohabitation des espèces de Zonitidae des grottes de la Roumanie au cadre des 5 provinces biospéologiques.

I

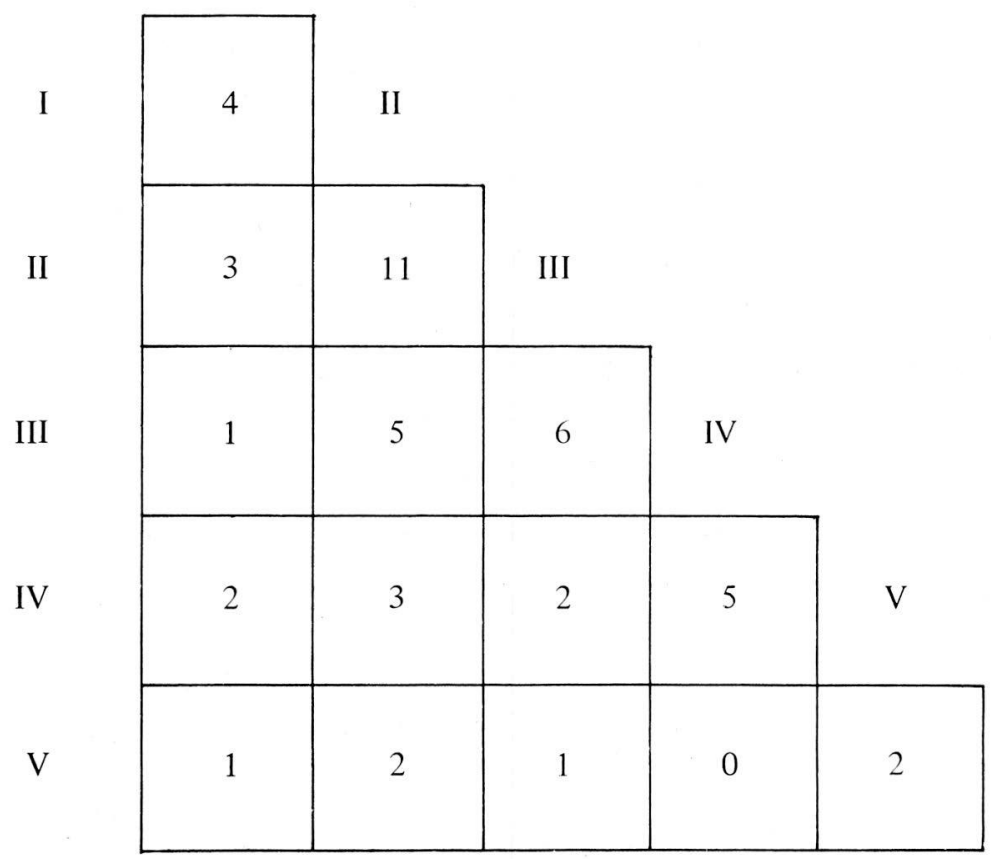

Nous pensons qu'il n'est pas sans intérêt de faire une brève analyse de la fréquence et l'abondance des 14 espèces cavernicoles de Zonitidae de Roumanie. Dans ce but, nous donnons dans le tableau 1 la fréquence des espèces dans les 5 provinces biospéologiques établies par Decou et Negrea (1969):

I. Les carpates orientales et mériodionales jusqu'à l'Olt;

II. Les Carpates méridionales entre l'Olt et le couloir Timis - Cerna;

III. Les monts du Banat;

IV. Les monts Apuseni;

V. La Dobrogea.

Dans ce tableau on a pris en considération toutes les grottes où les Zonitidae ont été signalées jusqu'à présent. Par "fréquence" nous comprenons le pourcentage de grottes positives pour l'espèce donnée du total des grottes à Zonitidae de la province respective. 
Il résulte du tableau que le plus grand nombre de grottes à Zonitidae existent dans le II $^{\mathrm{e}}$ province, la plus fréquente parmi les espèces étant Oxychilus glaber $(59,2 \%)$, suivie par O. depressus $(19,7 \%)$ et par Troglovitrea argintarui $(12,3 \%)$. C'est toujours l'espèce troglophile $O$. glaber qui est la plus fréquente aussi dans la III ${ }^{\mathrm{e}}$ province, c'es-à-dire dans les monts du Banat (68,7\%). Dans la province suivante (monts Apuseni) le pourcentage le plus élevé est détenu par $O$. depressus $(56,2 \%)$. On peut affirmer que ces deux espèces de Oxychilus sont les plus fréquentes dans les grottes de notre pays.

Dans le tableau 2 nous présentons le diagramme de la cohabitation des espèces de Zonitidae des grottes de Roumanie, dans le cadre des 5 provinces biospéologiques. Les chiffres à l'intersection de la même province représentent le nombre d'espèces de la province respective, et ceux de l'intersection de deux provinces différentes, le nombre d'espèces communes pour les deux provinces. Du diagramme il résulte que dans la $\mathrm{II}^{\mathrm{e}}$ province vivent la plupart des espèces et que les espèces communes les plus nombreuses existent dans les faunes des provinces II et III, ensuite dans celles des provinces I - II et II - IV.

En ce qui concerne l'abondance, on peut affirmer que les populations les plus riches en individus appartiennent aux espèces troglophiles Oxychilus glaber et $O$. depressus, qui sont en même temps aussi les plus fréquentes dans les provinces biospéologiques respectives. L'Espèce $O$. montivagus est mieux représentée numériquement seulement dans les 4 grottes du Banat. Les populations de Troglovitrea argintarui des 10 grottes d'Olténie n'étant pas très importantes cette espèce doit être protégée.

\section{RESUME}

Ce travail représente une contribution à l'étude des espèces de Zonitidae cavernicoles et endogées de Roumanie. Pour chacune des 24 espèces appartenant à 6 genres, on présente la distribution géographique, la répartition sur le territoire du pays et des données concernant les caractères morphologiques, l'appareil génital inclusivement. Le travail se termine par une brève analyse faunistique, zoogéographique et écologique.

\section{BIBLIOGRAPHIF}

BIELZ, E. A. (1863) - Fauna der Land- und Süsswasser-Mollusken Siebenbürgens. Hermannstadt, $V I$.

BIELZ, E. A. (1867) - Fauna der Land- und Süsswasser-Mollusken Siebenbürgens. Hermannstadt, VIII.

BOETGGER, C. (1940) - Zur Kenntnis der subterranen Molluskenfauna Sieben bürgens. Mededelingen van het Koninklijke Natuurhistorische Museum van België, Bruxelles, VXI.

DECOU, V., NEGREA, St. (1969) - Aperçu zoogéographique sur la faune cavernicole terrestre de Roumanie. Acta Zoologica Cracaviensia, XIV, 20.

FORCART, L (1959) - Taxionomische Revision paläarktischer Zonitidae, II. Anatomisch untersuchte Arten des Genus Aegopinella Lindholm. Arch. Moll., 88, 1/3. 
GROSSU, Al. (1955) - Gastropoda Pulmonata, in: Fauna Republicii Populare Române. Ed. Academiei, III, 1.

GROSSU, Al., RIEDEL, A. (1959) - Oxychilus deilus malinowskii (L. Pfeiffer, 1865) und die verwandten Formen. Arch. Moll., 87, 4/6.

GROSSU, Al., RIEDEL, A. (1968) - Beitrag zur Kenntnis der Zonitidae (Gastropoda) Rumäniens. Trav. Mus. Hist. Nat. "Grigore Antipa", VIII.

GUERGUIEV, V., BERON, P. (1962) - Essai sur la faune cavernicole de Bulgarie. Ann. Spéléol., XVII, 2-3.

HUDEC, Vl. (1964) - Zur Vorkommen der Schnecke Aegopinella epipedostoma (Fag.) und anderen Arten der Gattung Aegopinella Lindholm in der Tschechoslowakei. Acta Musei Nationalis Pragae, XX, 2.

HUSANU, O. (1961) - Contributiuni la cunoasterea gasteropodelor din Moldova, nota III. Anal. St. Univ. "Al. Cuza" Iasi (St. nat.), VII, 2.

HUSANU, O. (1965) - Contributiuni la studiul gasteropodelor din Moldova. Aegopinella Lindholm (Gastropoda, Zonitidae). Anal. St. Univ. "Al. Cuza" Iasi (St. nat.), XI, 2.

HUSANU, O. (1973) - Contributii la studiul gasteropodelor din Moldova. Studii si Comunicari, Muzeul de Stiinte Naturale, Bacau, 6.

KIMAKOWICZ, M. (1883) - Beitrag zur Mollusken-Fauna Siebenbürgens. Verh. Mitth. siebenb. Ver. Naturwiss., Hermannstadt, 33 .

KIMAKOWICZ, M. (1890) - Beitrag zur Mollusken-Fauna Siebenbürgens. II. Nachtrag. Verh. Mitth. siebenb. Ver. Naturwiss., Hermanstadt, 40.

MOTAS, C., DeCOU, V., BURGHELE, A. (1967) - Sur l'association pariétale des grottes d'Olténie (Roumanie). Ann. Spéléol., XXII, 3.

NEGREA, A. (1963) - Gasteropode (Mollusca) din fauna epigee si endogee a regiunilor carstice din R.P.R. Comun. Acad. R.P.R., XIII, 9.

NEGREA, A. (1965) - Gasteropode (Mollusca) din fauna regiunilor crstice din România. Lucr. Inst. Speol. "Emil Racovita", IV.

NEGREA, A. (1966) - Gasteropodele (Mollusca-Gastropoda) din pesterile României. Lucr. Inst. Speol. "Emil Racovita", V.

NEGREA, A., NEGREA, St. (1971) - Sur la synusie du guano des grottes du Banat (Roumanie). Trav. Inst. Spéol. "Emile Racovita", X.

NEGREA, A., RIEDEL, A. (1968) - Eine neue unterirdische Zonitiden-Art und Gattung (Gastropoda) aus Rumänien. Ann. Zool. Varsovie, XXVI, 5.

NEGREA, St., NEGREA, A. (1972) - Recherches sur l'association pariétale des grottes du Banat (Roumanie). Acta Zool. Cracoviensia, XVII, 3.

PINTER, L. (1972) - Die Gattung Vitrea Fitzinger, 1833 in den Balkanländern (Gastropoda: Zonitidae). Ann. Zool., Varsovie, XXIX, 8.

RIEDEL, A. (1957) - Revision der Zonitiden Polens (Gastropoda). Ann. Zool. Varsovie, XVI.

RIEDEL, A. (1961) - Uber Hyalina (Retinella) oscari Kimakowicz, 1883 - die typische Art der Untergattung Schistophallus A. J. Wagner, 1914 (Gastropoda, Zonitidae). Bull. Acad. Pol. Sci., Varsovie, 9.

RIEDEL, A. (1962) - Materialien zur Kenntnis der Paläarktischen Zonitidae (Gastropoda). Ann. Zool., Varsovie, 20.

RIEDEL, A. (1966) - Zonitidae (excl. Daudebardiinae) der Kaukasusländer (Gastropoda). Ann. Zool., Varsovie, XXIV, 1 .

RIEDEL, A. (1969) - Die Untergattungen Morlina A. J. Wagner und Riedelius Hudec der Gattung Oxychilus Fitzinger (Gastropoda, Zonitidae), Ann. Zool., Varsovie, XXVII, 6.

RIEDEL, A. (1972) - Die Untergattung Schistophallus A. J. Wagner in Europa und Kleinasien (Gastropoda, Zonitidae). Ann. Zool., Varsovie, XXIX, 7.

SOOS, L. (1917) - Zur Systematik und Anatomie der ungarichen Pulmonaten. Ann. Musei Nat. Hungarici, XV.

VANDEL, A (1964) - Biospéologie. La biologie des animaux cavernicoles. Ed. Gauthier-Villars, Paris.

ZAHARESCU, V. (1962) - Contributii la cunoasterea gasteropodelor terestre si de apa dulce din Moldova. Stud. Cercet. st., Biol., St. agric., Iasi, 13.

ZILCH, A., JAECKEL, S. (1962) - Die Weichtiere (Mollusca) Mitteleuropas, in: Die Tierwelt Mitteleuropas, II. 1. 